\title{
Convenient Optics for High Dispersion Small Angle Electron Diffraction with Highly Coherent Low Dose Illumination
}

\author{
Masahiro Kawasaki ${ }^{1}$ and Makoto Shiojiri ${ }^{2}$ \\ 1. JEOL USA Inc., 11 Dearborn Road, Peabody, MA, USA. \\ 2. Kyoto Institute of Technology, Kyoto, Japan. Present address: 1-297 Wakiyama, Kyoto, Japan
}

Structural analysis in the broad range of micrometer to nanometer or even to the atomic order is indispensable to understand the physical properties of functional materials and devices. Conventional transmission electron microscopy (TEM), scanning electron microscopy (SEM), small angle X-ray diffraction and small angle neutron diffraction are available for such analysis. As well-known, the small angle $\mathrm{X}$-ray diffraction is performed for various materials such as alloys, polymers, colloids and biomolecules including proteins and lipids. The small angle neutron scattering is performed for these substances as well as magnetic materials such as magnetic tapes, iron and steels, and rare-earth magnets, using unique characteristics of stronger scattering power for hydrogen and some light elements, higher penetration and magnetic scattering of neutron. By these diffraction techniques, the averaged structural parameters such as size, shape, interface, radial distribution etc. are obtained over an area irradiated by the incident beam in the bulk specimens, whose thickness is up to several ten $\mu \mathrm{m}$. Specimens in liquid can be observed as well. In contrast, TEM and SEM easily visualize the local structures through the thin films or on the surface of the bulk specimens. Environmental TEM and SEM can provide the structural information from the samples in liquid. The average information can be obtained by First-Fourier transformation (FFT) of an obtained image. It, however, is difficult to take TEM image for the radiation sensitive specimens. Electron diffraction (ED) patterns can be more easily obtained than TEM images with a significantly reduced dose, and the small angle electron diffraction technique provides similar information to that obtained by the small angle X-ray diffraction technique.

If we assume a lattice with a spacing $d$ as a periodical structure and a camera length $L$ for the ED, the diffraction peak of the 1 st order is observed at a distance of $r$ from the center primary peak on the detection screen, where $r \cong \lambda L / d$ and $\lambda$ is the wavelength of the electron. When $\lambda=0.00251 \mathrm{~nm}$ (for the incident electron beam of $200 \mathrm{KV}$ ) and $L=60 \mathrm{~m}$, we obtain $r=0.15 \mathrm{~mm}$ and the scattering angle $2.5 \mathrm{x}$ $10^{-6} \mathrm{rad}$ for $d=1 \mu \mathrm{m}$. An electron microscope with a coherent beam that is provided by a field emission gun can resolve the diffraction spots with high dispersion with an angular range of an order of $10^{-6} \mathrm{rad}$. In ordinary electron microscopes, however, the camera length choices are limited among the default camera lengths, especially for the high dispersion electron diffraction mode with a camera length of up to $8 \mathrm{~m}$ or so. They are only available in the low magnification mode where the objective lens is turned off automatically whereas the objective mini-lens is turned on as an objective lens. When the mode is switched, it doesn't keep the illumination condition which thereby is completely changed from what has been set in the imaging mode. Therefore, we describe here convenient optics for high dispersion electron diffraction with a highly coherent illumination that can be used for an extra long camera length of several tens of meters. In this method, the long camera length is obtained in such a way that a highly excited objective mini-lens transfers the diffraction formed at the back focal plane of the objective lens to the front of the $1^{\text {st }}$ intermediate lens, where the first image is usually formed, as shown in Fig. 1 left. Since the following set of projector lenses (the intermediate lenses 1 to 3 and the projector lens) is programed to magnify the image formed in front of the intermediate lens 1 , the diffraction pattern thus formed there by the objective mini-lens can be magnified accordingly (Fig. 1 right) with the preset lens program up to 
the maximum magnification $(\sim 1.5 \mathrm{M})$ on the film plane. It will be magnified more on the data-recording camera below the film chamber to the order of $200 \mathrm{~m}$ and even more at the end of the Gatan energy filter that provides energy filtering to the diffraction pattern.

A JEM-2100F with a Schottky gun and a Cryo pole-piece $(\mathrm{Cs}=2.0 \mathrm{~mm})$ for the objective lens providing the maximum magnification of $1 \mathrm{Mx}$ and a Gatan OneView camera (a pixel size of $15 \mu \mathrm{m}$ ) are used for a demonstration. Fig. 2 shows an image of a carbon replica of the 2D optical diffraction grating obtained with a dose rate of 39.3 electrons $/ \mathrm{nm}^{2}$ on the specimen. The following diffraction patterns were obtained under the same illumination condition. The replica carbon film was pre-shadowed by evaporating Au-Pd. The spacing of the gratings $d$ is $0.5 \mu \mathrm{m}$ and the slit width is $0.4 \mu \mathrm{m} \times 0.4 \mu \mathrm{m}$. Fig. 3 shows an electron diffraction pattern taken with a preset magnification of 10,000 where a camera length of $1.4 \mathrm{~m}$ is estimated. The diffraction rings from the Au-Pd evaporated film appear. Fig. 3 illustrates a small angle electron diffraction pattern of the diffraction grating, which was taken with a camera length as long as $140 \mathrm{~m}$ at a magnification of $1 \mathrm{M}$. The diffraction peaks corresponding to the spacing of gratings appear at the maxima of the interference function, modulated by the structure factor related with the slit width or grating width. The illumination angle was estimated $\sim 1 \times 10^{-6}$ rad. Thus, we have invented a potential technique, the small angle electron diffraction, for structure analysis of materials with a high coherent and low dose illumination.

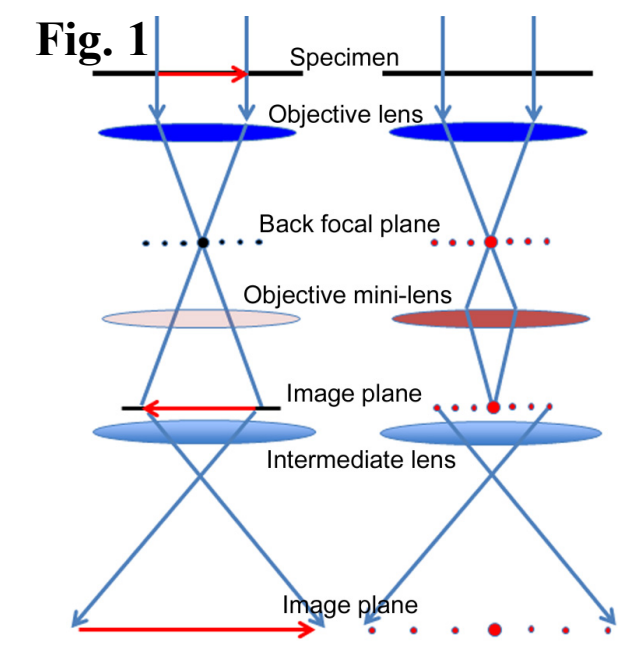

\section{Fig. 3}
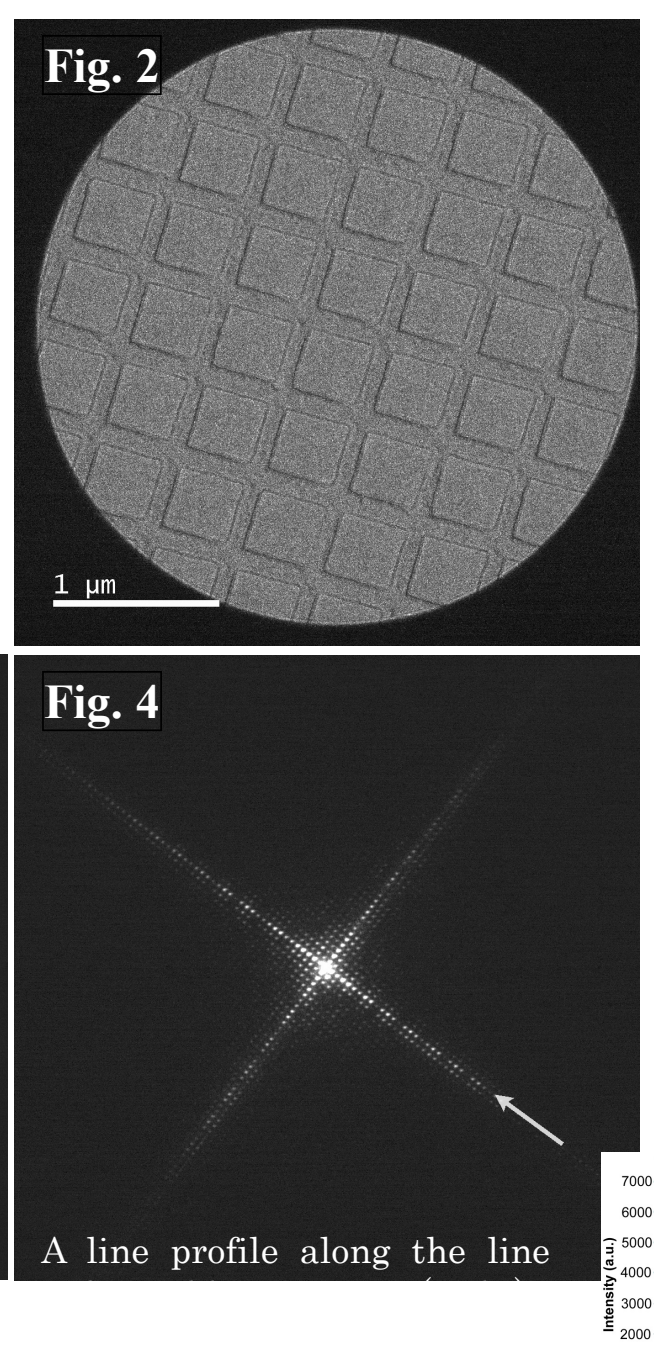

Figure 1. Ray diagrams for ordinary imaging (left) and the present small angle electron diffraction (right).

Figure 2. A parallel illumination on a grating sample set for demonstration in the present experiment including 7 slits in diagonal axes.

Figure 3. An electron diffraction pattern taken with the objective mini-lens (10 $\mathrm{Kx}$ )

Figure 4. A small angle electron diffraction pattern with the objective mini-lens (1 $\mathrm{Mx})$. The scattering angle between two spots is corresponding to $0.5 \mu \mathrm{m}$ in the real space. 